\title{
Inhibition of cyclooxygenase-2 prevents intra-abdominal adhesions by decreasing activity of peritoneal fibroblasts
}

This article was published in the following Dove Press journal:

Drug Design, Development and Therapy

15 June 2015

Number of times this article has been viewed

\author{
Guangbing Wei' \\ Xin Chen ${ }^{2}$ \\ Guanghui Wang' \\ Pengbo Jia ${ }^{1,3}$ \\ Qinhong $X \mathrm{u}^{2}$ \\ Gaofeng Ping' \\ Kang Wang' \\ Xuqi Li'
}

'Department of General Surgery, ${ }^{2}$ Department of Hepatobiliary Surgery, First Affiliated Hospital of Xi'an Jiaotong University College of Medicine, Xi'an, ${ }^{3}$ Department of General Surgery, First People's Hospital of Xianyang City, Xianyang,

People's Republic of China
Correspondence: Xuqi Li

Department of General Surgery, First Affiliated Hospital of Xi'an Jiaotong University College of Medicine, 277

West Yanta Road, Xi'an, Shaanxi 71006I,

People's Republic of China

Tel +862985323899

Fax +862985323899

Email lixuqi@।63.com
Background: Postoperative intra-abdominal adhesions are common complications after abdominal surgery. The exact molecular mechanisms that are responsible for these complications remain unclear, and there are no effective methods for preventing adhesion formation or reformation. The aim of the study reported here was to investigate the preventive effects and underlying potential molecular mechanisms of selective cyclooxygenase-2 (COX-2) inhibitors in a rodent model of postoperative intra-abdominal adhesions.

Materials and methods: The expression of COX-2 in postoperative intra-abdominal adhesions and normal peritoneal tissue was examined by immunohistochemistry and Western blot analysis. Assays were performed to elucidate the effect of COX-2 inhibition on hypoxia-induced fibroblast activity in vitro and on intra-abdominal adhesion formation in vivo.

Results: Hypoxia-induced COX-2 expression in peritoneal fibroblasts was increased in postoperative intra-abdominal adhesions. Inhibition of COX-2 attenuated the activating effect of hypoxia on normal peritoneal fibroblasts in vitro. Data indicate that selective COX-2 inhibitor prevents in vivo intra-abdominal adhesion by inhibition of basic fibroblast growth factor and transforming growth factor-beta expression, but not through an antiangiogenic mechanism. Furthermore, using selective COX-2 inhibitors to prevent intra-abdominal adhesions did not adversely affect the weight, bowel motility, or healing of intestinal anastomoses in a rat model.

Conclusion: These results show that hypoxia-induced COX-2 expression in peritoneal fibroblasts is involved in the formation of intra-abdominal adhesions. Inhibition of COX-2 prevents postoperative intra-abdominal adhesions through suppression of inflammatory cytokines.

Keywords: postoperative adhesions, COX-2, hypoxia, COX-2 inhibitors

\section{Introduction}

The development of postoperative intra-abdominal adhesions is one of the most common complications after abdominal surgery. Approximately 95\% of patients undergoing abdominal surgery will develop adhesions. ${ }^{1}$ Although adhesions are part of the wound-healing process, they may result in small bowel obstruction, postoperative abdominal pain, infertility, and other serious complications. ${ }^{2}$ About $15 \%$ of patients with adhesions develop bowel obstructions and require lysis, with a resulting mortality of 5\%-20\% and a high rate of recurrence. ${ }^{3}$ Thus, postoperative intra-abdominal adhesions represent a significant potential risk of additional complications. Accordingly, adhesions are a difficult problem for the surgeon and represent a significant public health cost. ${ }^{4,5}$ However, the exact molecular mechanisms by which this complication occurs remain unclear. ${ }^{6}$ At present, there are no effective methods for preventing adhesion formation. ${ }^{7,8}$ 
The peritoneum is the serous membrane that covers most of the intra-abdominal organs and is composed of a layer of mesothelial cells with sub-mesothelial tissue that contains plentiful fibroblasts. ${ }^{9}$ Surgical injuries to the peritoneal surface can result in adhesion formation, as a type of wound healing. The processes that result in either adhesion formation or normal peritoneal tissue repair are dependent on the function of fibroblasts. ${ }^{6}$ These cells have multiple functions, such as extracellular matrix (ECM) reorganization, collagen synthesis, and wound contraction. ${ }^{10}$

Following surgical injury to the peritoneum, inflammatory reactions at injury sites can result in the release of protein-enriched serosanguineous fluid and exudates, which cause congealing of the proteinaceous mass. If this congealed mass is not absorbed 3 to 5 days after formation, it will provide a scaffold for fibroblast proliferation and migration from underlying tissues, which can result in ECM deposition and the development of persistent adhesions. ${ }^{11}$

Hypoxia, resulting from tissue injury, appears to play a role in the pathophysiology of wound healing and adhesion formation. ${ }^{12}$ Induction of inflammatory markers and ECM proteins in normal peritoneal fibroblasts occurs in response to hypoxia. ${ }^{12,13}$ Moreover, fibroblasts from adhesions have been found to express cyclooxygenase-2 (COX-2), while normal peritoneal fibroblasts do not. Exposure of normal peritoneal fibroblasts to hypoxia induces COX-2 expression to levels seen in adhesion fibroblasts, ${ }^{14}$ indicating inhibition of COX-2 may provide the opportunity to reduce postoperative adhesion formation, as COX-derived prostaglandins (PGs) have also been implicated in adhesion formation.

Several COX-2 inhibitors have been shown to have potent ability to prevent intra-abdominal adhesions in small animals. However, the precise mechanism by which this occurs remains poorly understood. ${ }^{15-19}$ The aim of the study reported here was to investigate the role of COX-2 in postoperative intra-abdominal adhesions and explore the preventive effects and underlying potential molecular mechanisms of selective COX-2 inhibitors in a rodent model of adhesions.

\section{Materials and methods}

\section{Human tissue collection}

As previously described, ${ }^{20}$ a small piece of normal parietal peritoneal tissue from the anterior abdominal wall, lateral to the midline incision, or adhesion tissue was removed from patients who underwent laparotomy at the First Affiliated Hospital of the Medical College of Xi'an Jiaotong University. The latter excision was performed at the initiation of the surgery, after entry into the abdominal cavity. All patients gave informed written consent to tissue collection, and the protocol was approved by the Ethics Committee of Xi'an Jiaotong University.

\section{Fibroblast isolation and culture}

As described previously, ${ }^{21}$ harvested tissue samples were immediately placed in Dulbecco's Modified Eagle's Medium containing $10 \%$ fetal bovine serum, $200 \mu \mathrm{g} / \mathrm{mL}$ ampicillin, and $200 \mu \mathrm{g} / \mathrm{mL}$ streptomycin. Tissues were cut into small pieces and transferred into a sterile T-25 flask with dispase solution $\left(\right.$ Gibco $^{\circledR}$, Thermo Fisher Scientific, Waltham, MA, USA), and then incubated overnight at $37^{\circ} \mathrm{C}$. After centrifugation for 5 minutes at $1,400 \times g$, the samples were transferred to culture dishes in a 1:1 (v/v) mixture of Dulbecco's Modified Eagle's Medium/Ham's F12. The cultures were incubated at $37^{\circ} \mathrm{C}$ in a humidified atmosphere with $5 \% \mathrm{CO}_{2}$ for approximately 2 weeks. The selective COX-2 inhibitors celecoxib and valdecoxib (Sigma-Aldrich Co, St Louis, MO, USA) were dissolved in dimethyl sulfoxide (DMSO; Sigma-Aldrich $\mathrm{Co}$ ) and then added into the culture medium for experiments as previously reported. ${ }^{22,23}$

\section{Hypoxia conditions in cell culture}

To evaluate the effects of hypoxia, cells were incubated under normoxic conditions until 65\%-70\% confluence and subsequently cultured in $2 \% \mathrm{O}_{2}$ hypoxic conditions in a trigas incubator (HF100; Heal Force Development Ltd, Hong Kong, People's Republic of China), as described previously. ${ }^{24}$ Control cultures were placed in normoxic conditions. Cells were harvested at the 24-hour time point. All experiments were repeated three times.

\section{Western blot analysis}

Western blot analyses were performed as described previously. ${ }^{25}$ Total protein was extracted by mammalian protein lysis buffer (Thermo Fisher Scientific). Equivalent amounts of protein were subjected to sodium dodecyl sulfate-polyacrylamide gel electrophoresis and transferred to polyvinylidene fluoride membranes (EMD Millipore, Billerica, MA, USA). The membranes were incubated with the following primary antibodies overnight at $4{ }^{\circ} \mathrm{C}$ : anti-COX-2 antibody (Santa Cruz Biotechnology Inc, Dallas, TX, USA; 1:200 dilution), anti-COX-1 antibody (Santa Cruz Biotechnology Inc; 1:500 dilution), anti-hypoxia inducible factor-1 alpha (HIF-1 $\alpha$ ) antibody (Cell Signaling Technology, Inc, Danvers, MA, USA; 1:800 dilution), anti-vascular endothelial growth factor (VEGF) antibody (Santa Cruz Biotechnology Inc; 1:400 dilution), and anti-beta-actin ( $\beta$-actin) antibody (Santa Cruz Biotechnology 
Inc; 1:1,000 dilution). The blots were detected by a secondary antibody coupled to horseradish peroxidase (HRP; Santa Cruz Biotechnology Inc) and an enhanced chemiluminescence system (EMD Millipore). The density of specific protein bands was determined by Image-Pro Plus software (v 5.0; Media Cybernetics, Inc, Rockville, MD, USA).

\section{Quantification of prostaglandin $E_{2}$}

According to the manufacturer's instructions, prostaglandin $\mathrm{E}_{2}\left(\mathrm{PGE}_{2}\right)$ concentrations in cell culture supernatant were quantified using a $\mathrm{PGE}_{2}$ enzyme-linked immunosorbent assay kit (R\&D Systems, Inc, Minneapolis, MN, USA) after the number of cells was normalized to $0.5 \times 10^{6} / \mathrm{mL}$ in six-well culture plates.

\section{Real-time reverse-transcription polymerase chain reaction}

Total RNA was extracted from cells using TRIzol reagent (Invitrogen) and reverse transcribed using the PrimeScript ${ }^{\mathrm{TM}}$ RT Reagent Kit (TaKaRa, Dalian, China), according to the manufacturer's instructions. The mRNA levels were measured using the SYBR ${ }^{\circledR}$ Green Real-Time PCR (polymerase chain reaction) Master Mix with PCR primer sequences shown in Table S1. Glyceraldehyde 3-phosphate dehydrogenase (GAPDH) was used as the internal housekeeping gene control. Real-time reverse-transcription PCR was performed using an iQ5 Multicolor Real-Time PCR Detection System (Bio-Rad Laboratories Inc, Hercules, CA, USA). Fold changes in gene expression were quantified based on the ratio of target gene/ GAPDH mRNA using the $2-\Delta \Delta C$ t method. ${ }^{26}$ The following PCR conditions were used: 5 seconds at $94^{\circ} \mathrm{C}$, followed by 40 cycles at $94^{\circ} \mathrm{C}$ for 30 seconds, $60^{\circ} \mathrm{C}$ for 30 seconds, and $72^{\circ} \mathrm{C}$ for 30 seconds.

\section{Immunohistochemistry}

Immunohistochemical staining was performed using a strept avidin-biotin complex (SABC) kit (Maixin Biotech. Co., Ltd, Fuzhou, People's Republic of China) according to the manufacturer's instructions. The tissue sections were incubated with primary antibodies for COX-1 (1:100 dilution), COX-2 (1:50 dilution), basic fibroblast growth factor (bFGF) (1:100 dilution), transforming growth factor-beta (TGF- $\beta$ ) (1:100 dilution), VEGF (1:100 dilution), and cluster of differentiation (CD) 34 (1:50 dilution) overnight at $4^{\circ} \mathrm{C}$, then incubated with the appropriate biotinylated secondary antibody for 30 minutes at room temperature, followed by 30 minutes of incubation with streptavidin peroxidase (Dako $\mathrm{LSAB}^{\mathrm{TM}}+\mathrm{HRP}$ kit). After rinsing, the results were visualized using 3,3'-diaminobenzidine (DAB) tetrahydrochloride and the slides were counterstained with hematoxylin.

\section{Cell proliferation assays}

Cell proliferation assays were performed with the 3-(4,5dimethylthiazol-2-yl)-2,5-diphenyltetrazolium bromide (MTT) assay as described previously. ${ }^{27}$ Cells treated with DMSO served as a control. At various time points, $20 \mathrm{~mL}$ of a $5 \mathrm{mg} / \mathrm{mL}$ solution of MTT (Sigma-Aldrich Co) was added to each well, and cells were incubated for an additional 4 hours at $37^{\circ} \mathrm{C}$. Following MTT incubation, $150 \mathrm{~mL}$ per well of DMSO was added and viable cells were counted by measuring absorbance at $490 \mathrm{~nm}$ using a spectrophotometer (Bio-Rad Laboratories Inc).

\section{Lentivirus COX-2 short hairpin RNA transfection}

The lentivirus vector short hairpin RNAs (shRNAs) were constructed and synthesized by Shanghai GeneChem Co, Ltd (Shanghai, People's Republic of China). The shRNA sequences targeting human COX-2 were: 5'-TAA ACA CAG TGC ACT ACA TAC TTA TCA AGA GTA AGT ATG TAG TGC ACT GTG TTT TTT TTT C-3' and 5'-TCG AGA AAA AAA AAC ACA GTG CAC TAC ATA CTT ACT CTT GAT AAG TAT GTA GTG CAC TGT GTT TA-3'. A lentiviral vector ( $\mathrm{pGCSIL-GFP)}$ was used as a negative control. The virus titers produced were approximately $10^{9}$ transducing $\mu / \mathrm{mL}$ medium. Cells $\left(0.2 \times 10^{6} /\right.$ well $)$ seeded in six-well plates were transfected with lentivirus according to the manufacturer's instructions. The cells were used for further experiments at 48 hours after transfection. COX-2 mRNA and protein levels were determined using reverse-transcription PCR and Western blotting, respectively.

\section{Animals and formation of experimental adhesion}

Male Sprague-Dawley rats weighing 200 to $250 \mathrm{~g}$ were purchased from the Animal Center at the College of Medicine, $\mathrm{Xi}$ 'an Jiaotong University and housed individually in cages under constant temperature $\left(22^{\circ} \mathrm{C} \pm 2^{\circ} \mathrm{C}\right)$ with a 12-hour lightdark cycle. The animals were allowed access to water and standard rat chow ad libitum. All animal experimental protocols were approved by the Animal Care and Use Committee of the College of Medicine, Xi'an Jiaotong University.

Animals were anesthetized by inhalation of methoxyflurane for all procedures. Prior to incision, the abdomen was shaved and skin sterilized with Betadine. As described previously, ${ }^{28}$ a $2-3 \mathrm{~cm}$ midline abdominal incision was made 
and approximately $2-3 \mathrm{~cm}^{2}$ of the anterior cecal surface and the abdominal wall facing the treated cecum were lightly abraded 40 times with a swab to generate petechial hemorrhages. The cecum was placed back into its original position. For the hyaluronan group, $1 \mathrm{~mL}$ of medical hyaluronan gel (Qingdao Haitao Biochemical Co., Ltd., Qingdao People's Republic of China) was daubed on the abraded area. The other group received no treatment. The abdomen was closed in two layers using interrupted 3-0 Vicryl ${ }^{\circledR}$ sutures. A sham operation group was prepared in the same manner, but without abrasion.

Celecoxib (40 mg/kg) was orally administered to the animals twice daily for 7 days as described previously. ${ }^{15}$ Parecoxib (1 mg/kg) was injected by the tail vein daily for 7 days as described previously. ${ }^{29}$ The other group of animals was given normal saline solution as a control.

\section{Adhesion evaluation}

Seven days after the surgery, the rats were anesthetized, and intra-abdominal adhesion formation was evaluated according to a modified classification system described by Katada et al. ${ }^{30}$ Assessment was performed by a single researcher blinded to the treatment data of the study. The tenacity was quantified based on the severity of adhesions, and assigned to one of five categories: 0 (no adhesion), 1 (mild, localized, and easy to separate), 2 (moderate), 3 (severe, very widespread, and difficult to separate), and 4 (the most severe). The extent was quantified as a percentage of abrasion with adhesions present and assigned to one of five categories: 0 (no adhesion), 1 ( $\leq 25 \%), 2$ (26\%-50\%), 3 (51\%-75\%), and $4(\geq 75 \%)$. The area was scaled as the number of tissues involving adhesion and assigned to one of five categories: 0 (no adhesion), 1 (only the tissues with abrasion), 2 (one other tissue, including the small intestine, liver, omentum, and spleen), 3 (two other tissues), and 4 (three or more other tissues). The final scores ranged from 0 to 12 .

\section{Picrosirius red staining for collagen}

Picrosirius red staining for collagen was achieved using $0.1 \%$ picrosirius red (Direct Red 80; Sigma-Aldrich Co) and counterstained with Weigert's hematoxylin (Wako Pure Chemical Industries, Ltd., Osaka, Japan). The percentage of positively stained areas was evaluated by Image-Pro Plus software (Media Cybernetics, Inc., Bethesda, MD, USA) in eight randomly selected fields, and the average of the eight values was taken as the collagen content in the adhesions.

\section{Hydroxyproline determination}

Hydroxyproline content was determined by hydroxyproline assay kit (Sigma-Aldrich Co) according to the manufacturer's instructions. Tissue hydroxyproline levels, which were used as an indicator of adhesion severity, were presented as micrograms of hydroxyproline per gram of protein.

\section{Measurement of gastrointestinal transit, anastomotic bursting pressure, and cotton pellet granuloma}

Rats were divided randomly into four groups $(n=8$ per group). Operations were performed as described previously. ${ }^{31}$ Briefly, all animals underwent general anesthesia; laparotomy; descending intestinal resection $(15 \mathrm{~cm}$ proximal to the ileocecal junction); and subsequent anastomosis using eight single-layer, everting, interrupted 6/0 monofilament biodegradable sutures. Sterile cotton pellets $(30 \pm 3 \mathrm{mg})$ were implanted intraperitoneally in the rats as described by Fu et al. ${ }^{32}$ The animals then received treatment similar to the rat adhesion formation model. Rats were fed a standard diet and allowed water ad libitum. They were checked for wound healing and passage of stool.

On the 7 th day, each rat was administered by gavage a $0.2 \mathrm{~mL}$ solution of $1.5 \%$ carboxy methylcellulose sodium salt and 5\% charcoal as a marker. After 30 minutes, the rats were sacrificed and the cotton pellet removed, dried overnight at $70^{\circ} \mathrm{C}$, and weighed. The increased weight of the pellet was taken to be a measure of the weight of granulomas. The gastrointestinal tract was removed. The distance traveled by the charcoal plug from the pylorus to the cecum was determined and expressed as a percentage of the total length of the small intestine. The anastomotic segments approximately $4 \mathrm{~cm}$ in length (with the anastomosis in the middle) were resected. The anastomotic bursting pressure was quantified as previously described. ${ }^{31}$

\section{Statistical analyses}

The results are expressed as the mean \pm standard error or the median. An independent Student's $t$-test was performed to analyze the statistical significance between two groups. Differences among groups were evaluated by Kruskal-Wallis variance analysis, followed by the posthoc Mann-Whitney $U$ - test with SPSS software (v 13.0). $P<0.05$ was considered statistically significant.

\section{Results \\ Expression of HIF-I $\alpha$, COX-I, COX-2, and VEGF in peritoneal adhesion tissues}

To determine the possible role of hypoxia-induced COX-2 expression or angiogenesis in intraperitoneal adhesion 
formation, the expression of HIF-1 $\alpha$, COX-1, COX-2, and VEGF in the surgical cases was explored using Western blotting (Figure 1A). The results show that HIF-1 $\alpha, \mathrm{COX}-2$, and VEGF were markedly increased in peritoneal adhesion tissues compared with in normal peritoneum. However, no significant changes were found in the expression of COX-1 protein between adhesion tissues and normal peritoneum. To evaluate COX-2 mRNA levels, real-time reverse-transcription PCR analysis was performed on 17 adhesive tissues and 20 normal peritoneum tissues. The relative level of COX-2 mRNA was increased in the adhesion tissues compared with in normal peritoneum tissues $(P<0.05)$ (Figure 1B). Previous studies ${ }^{30}$ have demonstrated that $\mathrm{PGE}_{2}$ is one of the primary $\mathrm{COX}$ 2-derived mediators responsible for adhesion formation. Therefore, we focused on $\mathrm{PGE}_{2}$ content in tissues, and found that $\mathrm{PGE}_{2}$ levels in peritoneal adhesions were higher than in normal peritoneum specimens (Figure 1C).

To further confirm these results, COX-2 expression in the surgical cases was explored. Intensive immunohistochemical staining of COX-2 was detected in the cytoplasm of fibroblasts in peritoneal adhesions, whereas there was only rare staining in normal peritoneal tissues (Figure 1D).

Overexpression of COX-2 was detected in $72.5 \%$ of peritoneal adhesion samples compared with in only $20 \%$ of normal peritoneum samples $(P<0.05)$ (Figure 1E). The results show that the levels of $\mathrm{COX}-2$ protein in peritoneal adhesion tissues were significantly elevated compared to in normal peritoneal tissues.

\section{Hypoxia increased COX-2 expression and activity in fibroblasts from the normal peritoneum to levels similar to those in adhesion fibroblasts}

To determine the effect of hypoxia on activation of primary fibroblasts from human normal peritoneum or adhesion fibroblasts, cells were cultured for 24 hours under hypoxic conditions and assessed for protein expression. The results show that hypoxia significantly increased HIF- $1 \alpha, \mathrm{COX}-2$, and VEGF levels in normal peritoneal fibroblasts to levels

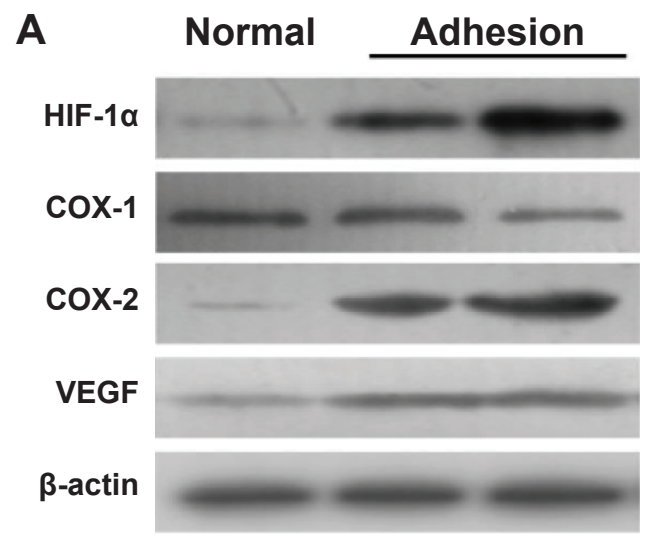

B

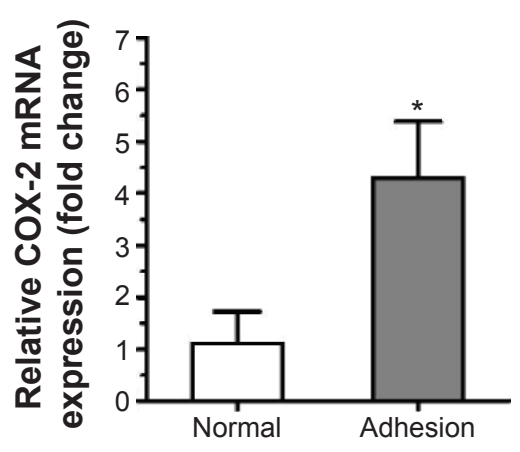

C

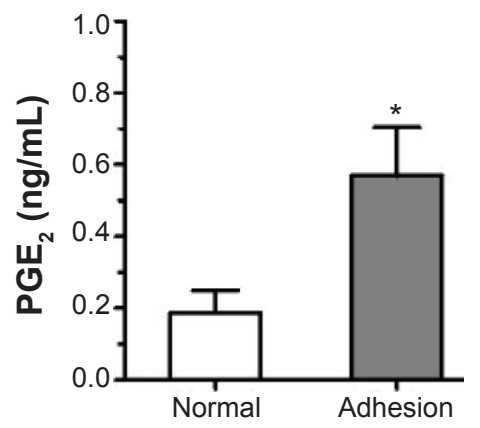

D

Normal

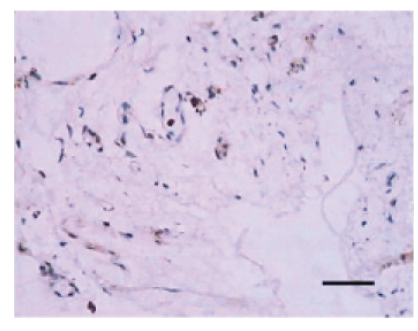

Adhesion

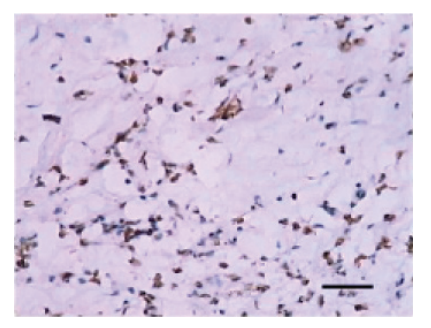

$\mathbf{E}$

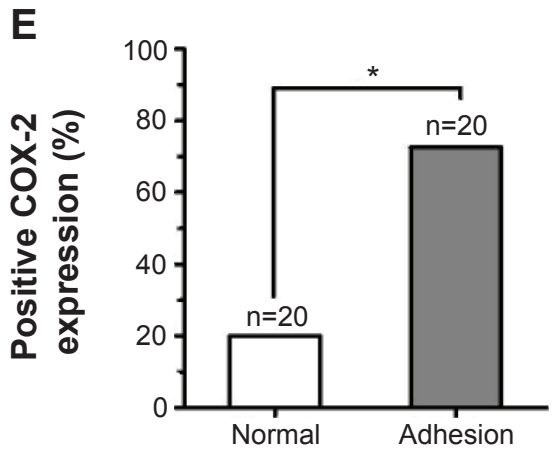

Figure I Expression of anti-hypoxia inducible factor-I alpha (HIF-I $\alpha$ ), cyclooxygenase (COX)-I, COX-2, and vascular endothelial growth factor (VEGF) in peritoneal adhesion tissues.

Notes: (A) Representative Western blot analyses showing HIF-I $\alpha$, COX-I, COX-2, and VEGF expression levels in normal and peritoneal adhesion tissues. (B) Real-time reverse-transcription polymerase chain reaction was used to quantify the relative levels of COX-2 mRNA. (C) Enzyme-linked immunosorbent assay analysis of prostaglandin $\mathrm{E}_{2}\left(\mathrm{PGE}_{2}\right)$ contents in normal and peritoneal adhesion tissues. (D) Representative immunohistochemistry images showing the overexpression of COX-2 in normal and peritoneal adhesion tissues. Compared with normal peritoneum tissues, peritoneal adhesion tissues displayed increased levels of COX-2 in fibroblasts. (E) The relative scores for immunohistochemical staining of COX-2 in normal and peritoneal adhesion tissues. $P$-values were calculated using the Mann-Whitney rank test. * $P<0.05$. (B) Columns, mean; bars, standard deviation. (C) Magnification I00x; scale bars $50 \mu \mathrm{m}$. 
similar to those in adhesion fibroblasts under normoxia. However, hypoxia had no effect on COX-1 expression (Figure 2A). Similar results were observed with COX-2 mRNA levels in normal and adhesion fibroblasts by realtime reverse-transcription PCR (Figure 2B). Furthermore, $\mathrm{PGE}_{2}$ levels in the culture medium showed that $\mathrm{PGE}_{2}$ was significantly increased in normal fibroblasts and slightly increased in adhesion fibroblasts under hypoxia (Figure 2C).
Immunofluorescence analysis revealed that COX-2 levels, which were paralleled by levels of alpha-smooth muscle actin ( $\alpha$-SMA), a molecular marker of activation, were more stable and predominantly expressed in normal fibroblasts under hypoxia compared to under normoxia (Figure 2D). These data suggest that hypoxia increases COX-2 expression and activity in fibroblasts from the normal peritoneum to levels similar to those in peritoneal adhesions.
A

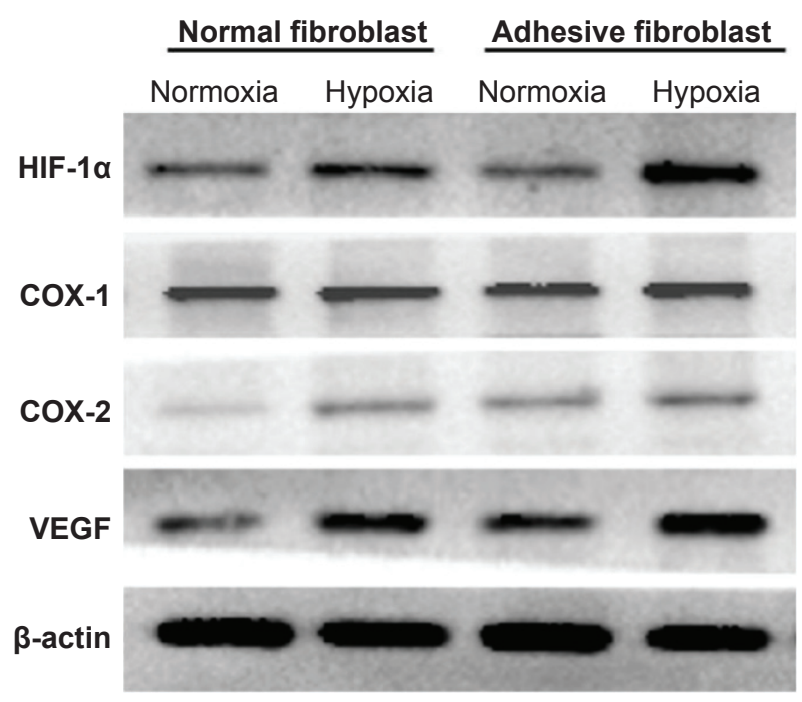

B

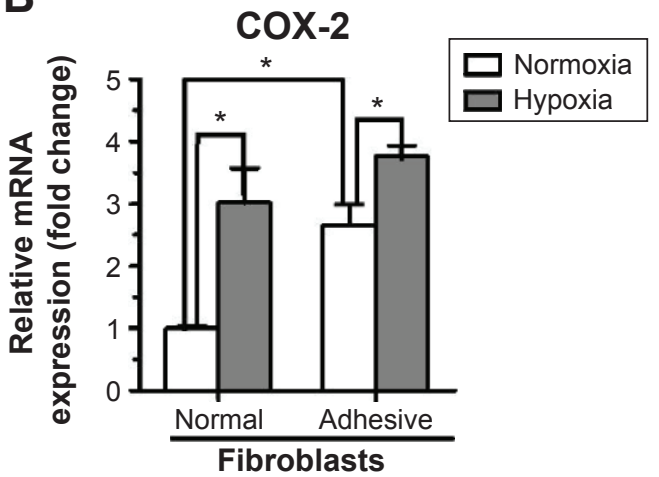

C

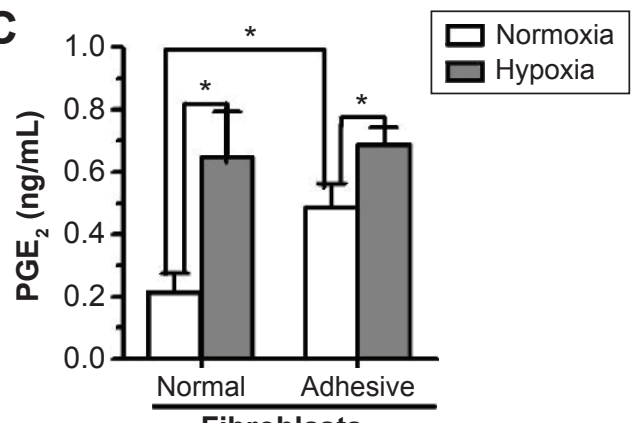

Fibroblasts

D a-SMA coX-2
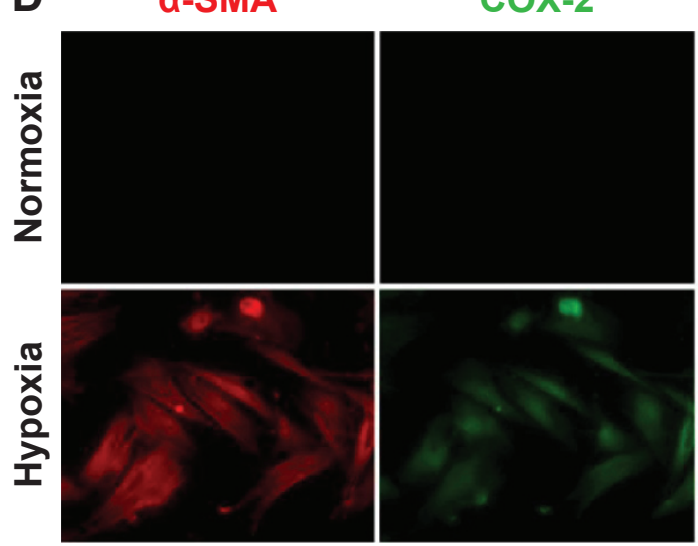

DAPI

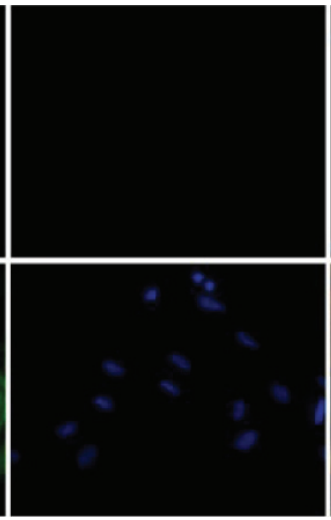

Merge

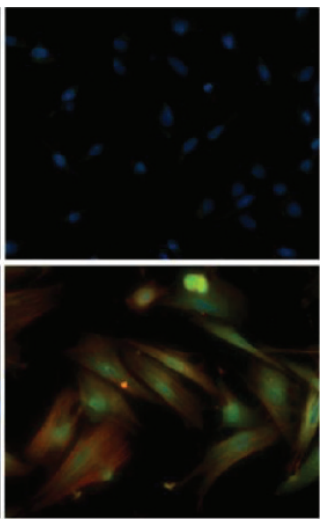

Figure 2 Hypoxia promotes cyclooxygenase (COX)-2 expression and function in fibroblasts from the normal peritoneum.

Notes: (A) Western blot analyses showing anti-hypoxia inducible factor-I alpha (HIF-I $\alpha$ ), COX-I, COX-2, and vascular endothelial growth factor (VEGF) expression levels in fibroblasts from normal peritoneum tissues in response to hypoxia. Beta-actin was used as an internal control. Hypoxia obviously increased HIF-I $\alpha$, COX-2, and VEGF expression in fibroblasts from normal peritoneal tissues in vitro. (B) Real-time reverse-transcription polymerase chain reaction was used to quantitate the relative levels of COX-2 mRNA. (C) Enzyme-linked immunosorbent assay analysis of prostaglandin $E_{2}\left(P_{G} E_{2}\right)$ contents in fibroblasts from normal peritoneal tissues in response to hypoxia. (D) The expression of alpha-smooth muscle actin ( $\alpha$-SMA) (red) and COX-2 (green) protein in fibroblasts from normal peritoneal tissues in response to hypoxia. Cellular nuclei were stained with 4',6-diamidino-2-phenylindole (DAPI) (blue). (B) Columns, mean; bars, standard deviation; $* P<0.01$. (C) *P<0.05. (D) Magnification 200×. 


\section{COX-2 inhibition decreases hypoxic activation of fibroblasts from the normal peritoneum or activated fibroblasts from adhesive tissue}

To further determine the role of COX-2 in the activation of fibroblasts from the normal peritoneum in response to hypoxia, a small interfering RNA (siRNA) specific for COX-2 in fibroblasts was used. Downregulation of COX-2 expression was confirmed using Western blotting (Figure 3A). Although the expression of HIF- $1 \alpha$ increased in both COX-2 siRNA and control siRNA cells under hypoxia, no significant changes were found in the expression of $\alpha$-SMA and VEGF under hypoxia in the COX-2 siRNA group (Figure 3A). In contrast, $\alpha$-SMA and VEGF levels were significantly elevated under hypoxia compared to under normoxia in the control siRNA group.

To explore the role of COX-2 on cell proliferation under normoxia and hypoxia, fibroblasts were seeded onto 96-well plates and at various time points the proliferative rates of fibroblasts were determined by MTT assay (Figure 3B). The results show that the proliferation of fibroblasts increased in response to hypoxia compared with in response to normoxia in the control siRNA group $(P<0.05)$. However, inhibition of COX-2 by siRNA completely inhibited the increased cell proliferation caused by hypoxia. Additionally, elevated $\mathrm{PGE}_{2}$ levels induced by hypoxia in cell culture supernatants were significantly decreased in the COX-2 siRNA group compared to in the control siRNA group $(P<0.05)$ (Figure 3C).

Two different selective COX-2 inhibitors were administered to fibroblasts in the presence of hypoxia. As shown in Figure 3D, $10 \mu \mathrm{M}$ celecoxib or valdecoxib, both highly selective COX-2 inhibitors, had negative effects on COX-2 expression. On the other hand, both celecoxib and valdecoxib reduced $\alpha$-SMA and VEGF expression in hypoxia-activated fibroblasts. Blockade of COX-2 activity by celecoxib and valdecoxib decreased cell proliferation under hypoxia similar to the inhibition of COX-2 expression by siRNA (Figure 3E). $\mathrm{PGE}_{2}$ levels in cell culture supernatants were significantly downregulated after 48 hours of celecoxib and valdecoxib treatment under hypoxia compared to in untreated controls (Figure 3F). Taken together, these findings strongly indicate that COX-2 inhibition decreases the hypoxic activation of fibroblasts from the normal peritoneum.

Valdecoxib significantly decreased COX-2, $\alpha$-SMA, and VEGF expression (Figure 3G), inhibited cell proliferation (Figure $3 \mathrm{H}$ ), and reduced $\mathrm{PGE}_{2}$ levels in adhesion fibroblasts (Figure 3I).
Selective COX-2 inhibitors prevent intraabdominal adhesions in a rat model

There were no visible differences in weight between the five groups and all animals appeared healthy. The control animals developed bowel-to-parietal peritoneum, omentumto-parietal peritoneum, bowel-to-bowel, and omentum-tobowel adhesions. Animals that underwent sham operation surgery barely developed any intra-abdominal adhesions. In contrast, the animals treated with selective COX-2 inhibitors (ie, celecoxib and parecoxib) had fewer intra-abdominal adhesions than controls. However, intra-abdominal adhesions were only slightly fewer in the animals treated with hyaluronan (Figure 4A).

Adhesion scores for the macroscopic classification were found to be significantly different between the five groups (Figure 4B). In the celecoxib group and parecoxib group, the macroscopic classification scores were lower than in the control or hyaluronan group. However, the parecoxib group had lowest adhesion scores of all groups. With regards to preventing adhesions, there was a significant difference between two COX-2 selective inhibitor treatment groups (celecoxib and parecoxib), the control group $(P<0.05)$, and the hyaluronan group $(P<0.05)$ (Figure $4 \mathrm{C})$.

As shown in Figure 4D and E, the collagen content of adhesions was significantly decreased in the celecoxib group and the parecoxib group compared to in the control group. Moreover, there were significant decreases in hydroxyproline levels in both COX-2 selective inhibitor treatment groups compared to in the control group $(P<0.05)$ and the hyaluronan group $(P<0.05)$ (Figure $4 \mathrm{~F})$. Both the macroscopic and objective data suggest that selective COX-2 inhibitors prevent intra-abdominal adhesions in the rat model.

\section{The effect of selective COX-2 inhibitors on intra-abdominal adhesion prevention possibly results from inhibition of bFGF and TGF- $\beta$ expression}

HIF- $1 \alpha$ expression in adhesion tissues was increased in the four groups that underwent mechanical injury, regardless of the interventions (Figure 5A). Compared with the control group, both celecoxib and parecoxib decreased COX-2 expression in peritoneal adhesion tissue (Figure 5A). COX-2 expression was only slightly decreased in the hyaluronan group. These results are consistent with the results of Western blotting and COX-2 mRNA levels (Figure 5B). Furthermore, mRNA levels of type I collagen and $\alpha$-SMA were significantly decreased by celecoxib or parecoxib 
A

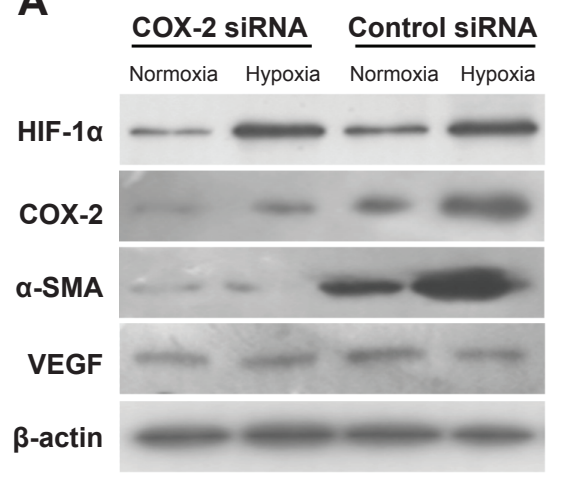

D

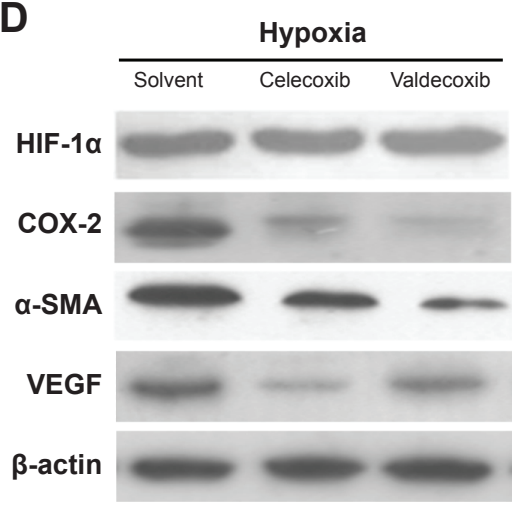

G

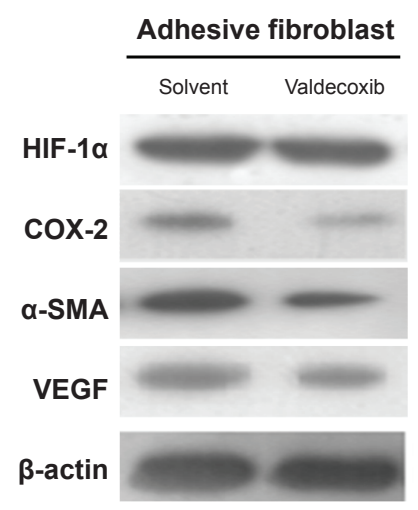

B

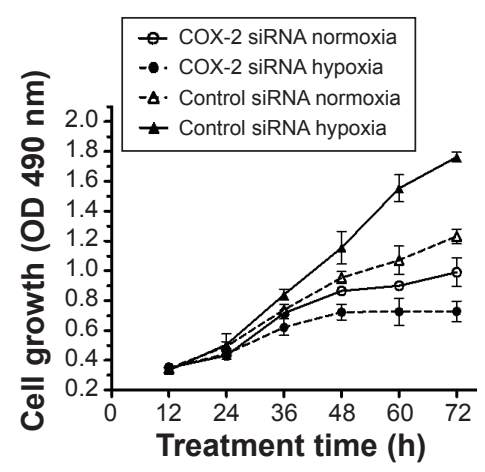

E

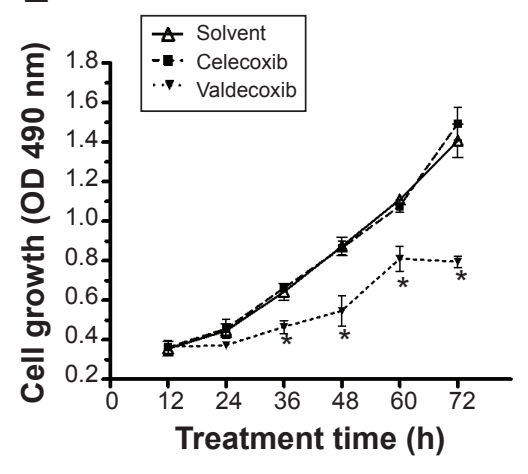

H

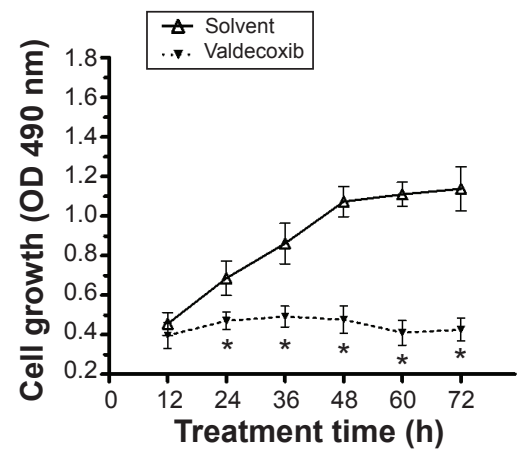

C

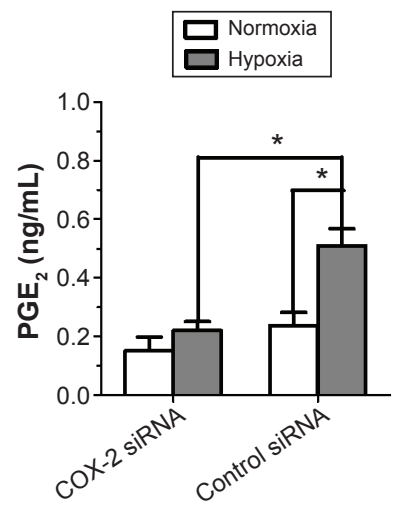

$\mathbf{F}$

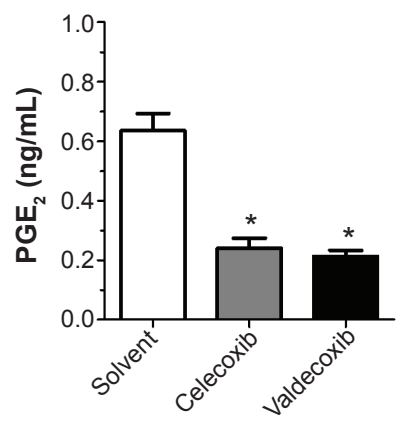

I

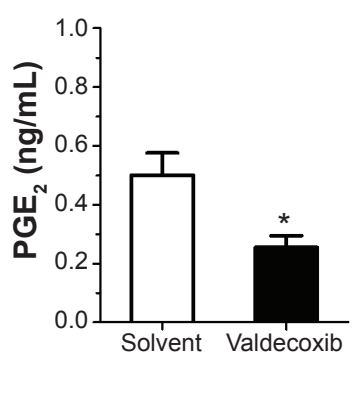

Figure 3 Cyclooxygenase (COX)-2 inhibition attenuates the activated effect of hypoxia on fibroblasts from normal peritoneum.

Notes: (A) Western blot analysis of the expression of the indicated proteins in fibroblasts in response to hypoxia with or without COX-2 silencing. Beta-actin was used as an internal control. (B) A cell proliferation assay using 3-(4,5-dimethylthiazol-2-yl)-2,5-diphenyltetrazolium bromide (MTT) showed the proliferation rate of fibroblasts in each group. COX-2 inhibition by small interfering RNA (siRNA) completely abrogated the accelerated cell proliferation caused by hypoxia. (C) Enzyme-linked immunosorbent assay (ELISA) analysis of prostaglandin $E_{2}\left(P_{2}\right)$ contents in fibroblasts in each group. The elevated $P G E_{2}$ levels induced by hypoxia in cell culture supernatants were significantly decreased in the COX-2 siRNA group. $* P<0.05$ (D) Western blot analysis of the expression of the indicated proteins in fibroblasts under hypoxia with or without COX-2 inhibitors. (E) A cell proliferation assay using MTT showed the proliferation rate of fibroblasts under hypoxia with or without COX-2 inhibitors. (F) ELISA analysis of PGE 2 contents in fibroblasts under hypoxia with or without COX-2 inhibitors. (G) Western blot analysis of the expression of the indicated proteins in adhesive fibroblasts with or without COX-2 inhibitors. (H) A cell proliferation assay using MTT showed the proliferation rate of adhesive fibroblasts with or without COX-2 inhibitors. (I) ELISA of PGE ${ }_{2}$ contents in adhesive fibroblasts with or without COX-2 inhibitors. ${ }^{*} P<0.05$.

Abbreviations: $\alpha$-SMA, alpha-smooth muscle actin; HIF-I $\alpha$, anti-hypoxia inducible factor-I alpha; OD, optical density; VEGF, vascular endothelial growth factor; h, hours.

treatment when compared with the control (Figure 5B). Using the presence of $\alpha$-SMA as a marker of fibroblast activation, we determined that $\alpha$-SMA was downregulated after celecoxib or parecoxib treatment compared with in the control and hyaluronan groups. These results provide additional evidence that the inhibition of intra-abdominal adhesion indeed involves fibroblast growth inhibition (Figure 5C).

Immunohistochemical analysis showed that the expression of COX-2 in rat intra-abdominal adhesion tissue was increased after treatment with the selective COX-2 inhibitors in the control group compared with the normal peritoneum in the sham 


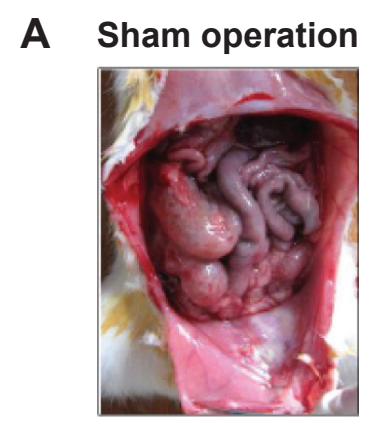

B

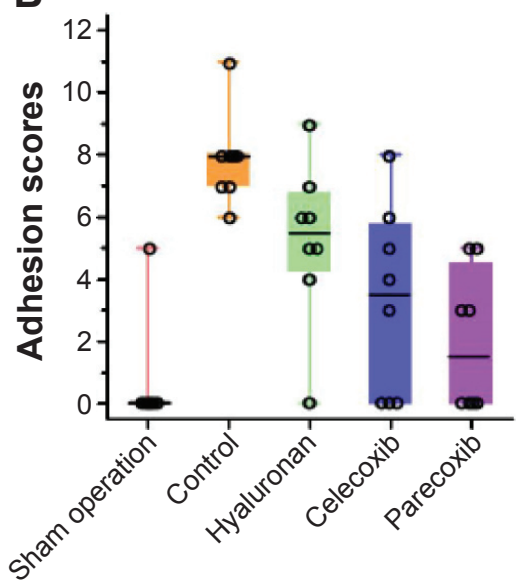

Hyaluronan
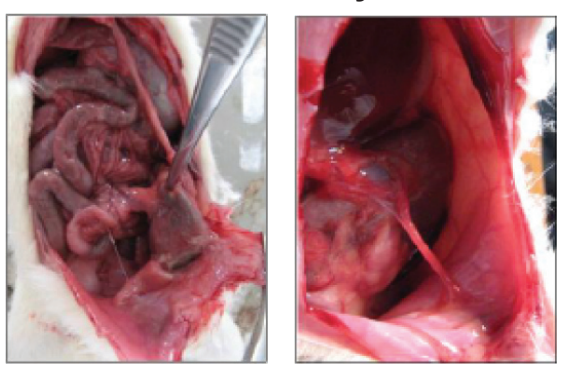

C
Celecoxib
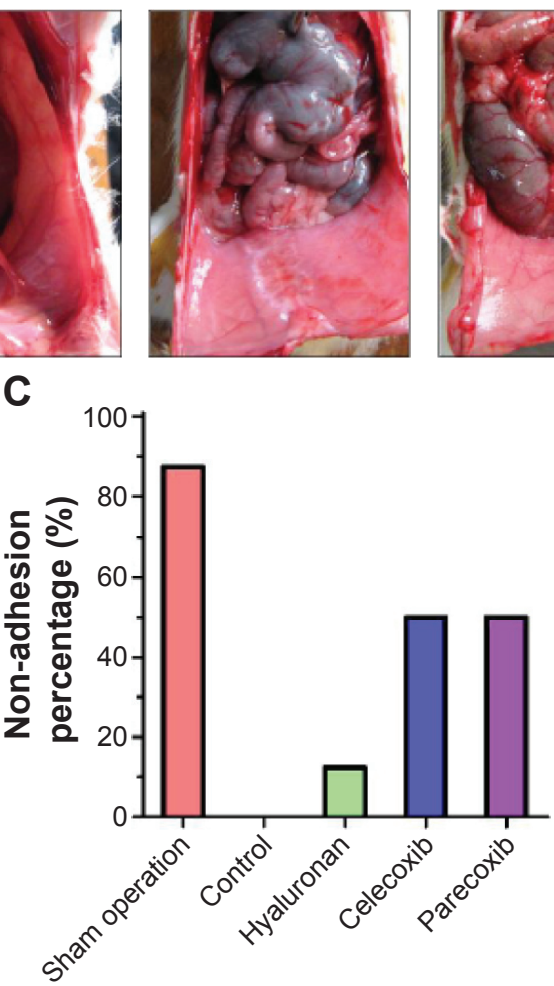

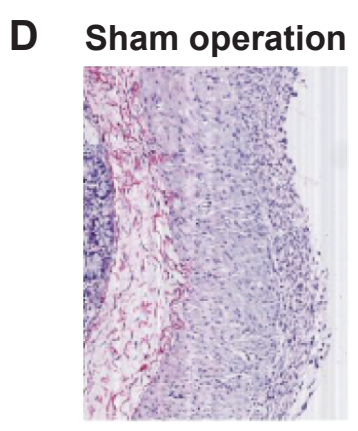

E

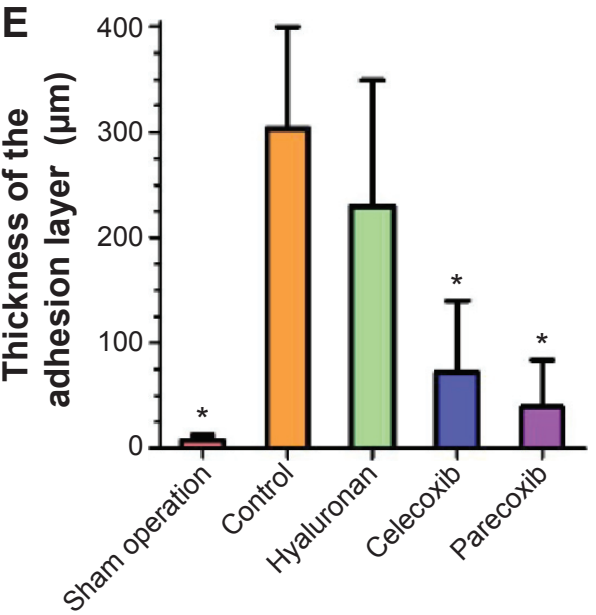

\section{Control}

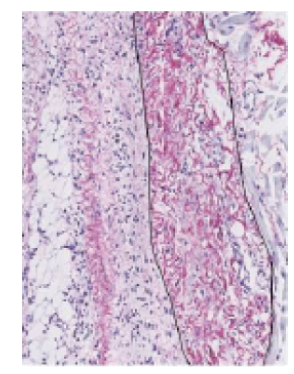

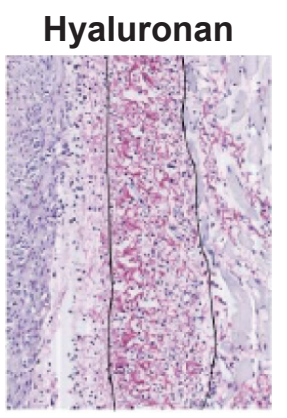
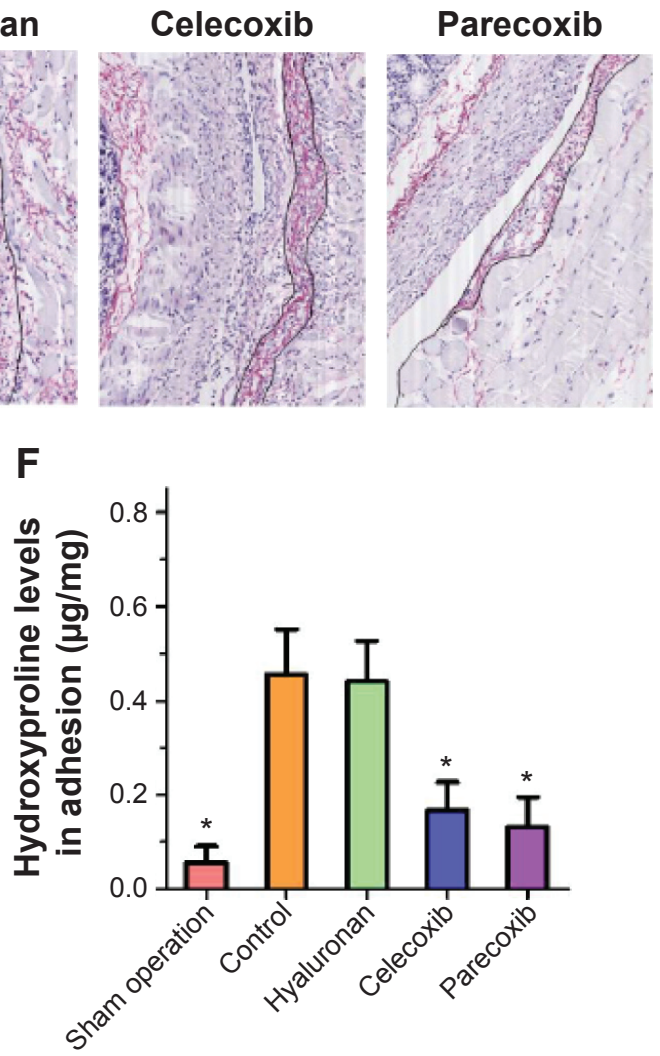

Figure 4 Cyclooxygenase (COX)-2 inhibition attenuates the activated effect of hypoxia on fibroblasts from normal peritoneum.

Notes: (A) Representative images of intra-abdominal adhesions in each group at day 7. (B) Adhesion scores for the macroscopic classification. The celecoxib and parecoxib treatment groups had the lowest scores of adhesion. (C) Non-adhesion incidence in each group. COX-2 selective inhibitor treatment groups (celecoxib and parecoxib) showed the most non-adhesion incidences compared with the control group or hyaluronan group. (D) Representative images of picrosirius red staining for collagen in tissue sections in each group. The adhesive tissue is between the two lines drawn in the images. (E) The collagen content in the adhesions was significantly decreased in the celecoxib group and the parecoxib group compared to in the control group. (F) There were significant decreases in hydroxyproline levels in both COX-2 selective inhibitor treatment groups when compared with the control group or hyaluronan group. $* P<0.05$. 

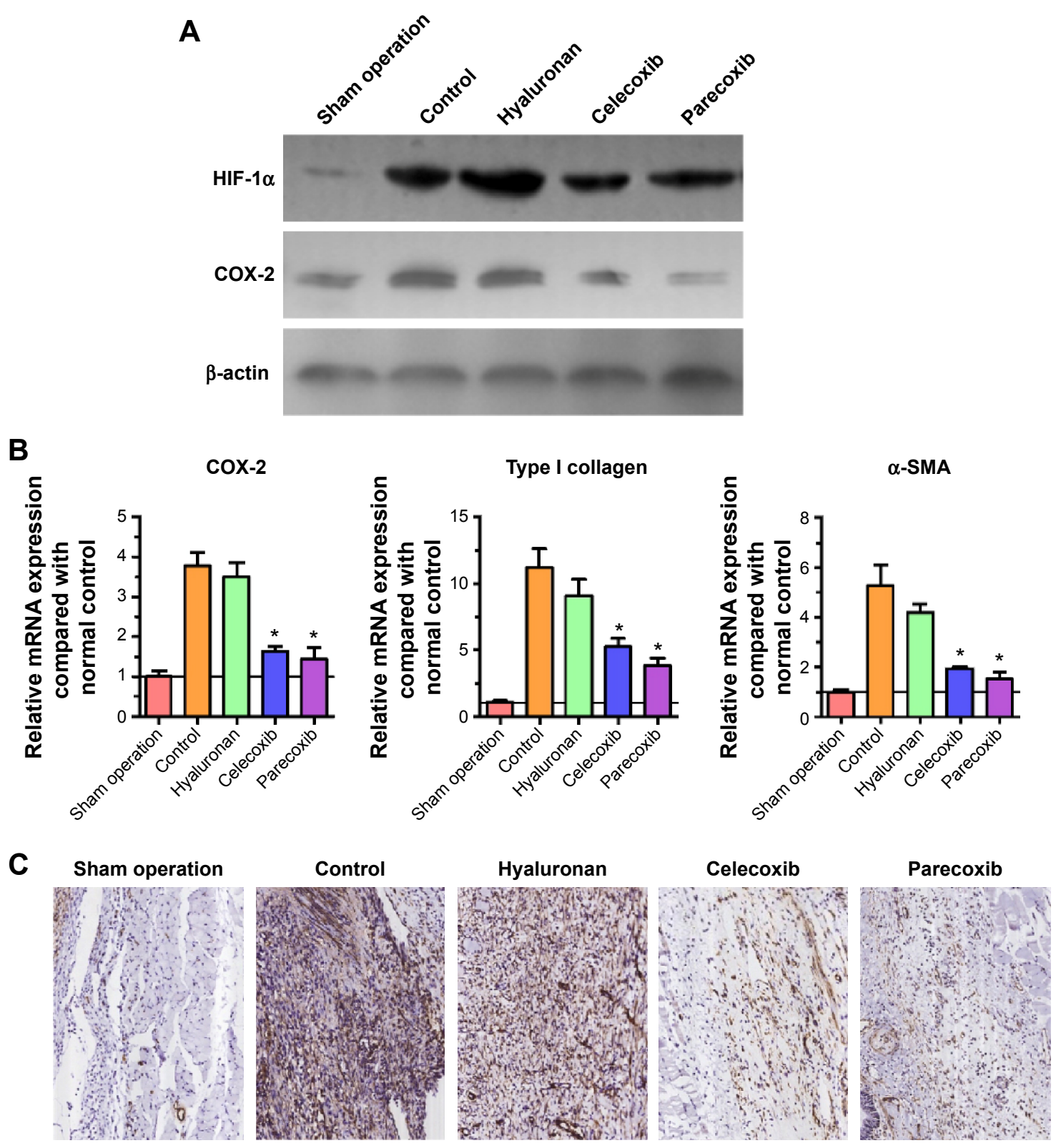

Figure 5 Selective cyclooxygenase (COX)-2 inhibitors decreased COX-2 expression in injured peritoneum.

Notes: (A) Western blot analysis showed that the either celecoxib or parecoxib obviously decreased COX-2 expression in injured peritoneum compared to adhesion tissue in the control group. (B) Real-time reverse-transcription polymerase chain reaction showed that mRNA expression of COX-2, alpha-smooth muscle actin ( $\alpha$-SMA), and type I collagen was significantly decreased as a result of celecoxib or parecoxib treatment compared to the control. (C) Immunohistochemical analysis of $\alpha$-SMA in intra-abdominal adhesion tissues from groups that underwent peritoneum and cecum abrasion, and normal peritoneum in the sham operation group. $* P<0.05$. Magnification $200 \times$.

Abbreviation: HIF-I $\alpha$, anti-hypoxia inducible factor-I alpha.

operation group (Figure 6A). The sections from peritoneal adhesions stained intensely positive for inflammatory cytokines bFGF and TGF- $\beta$ in the control group, confirming the presence of an inflammatory process (Figure $6 \mathrm{~B}$ and $\mathrm{C}$ ). The expression of $\mathrm{bFGF}$ and TGF- $\beta$ were significantly lower in both the celecoxib and parecoxib treatment groups than in the control group and the hyaluronan group.

Because a previous study ${ }^{15}$ claimed that post-operation intra-abdominal adhesion formation was possibly dependent on angiogenesis, immunohistochemical staining for a vital angiogenesis factor, VEGF, and an endothelial cell marker, CD34, was performed to identify new blood microvessels. In the control group sections, the expression of VEGF was significantly higher than in those of the sham operation group, which had negative or very low levels (Figure 6D). However, compared with the control group, the level of VEGF expression was not changed in the parecoxib treatment group, but was decreased in the celecoxib treatment group (Figure 6D). 
A

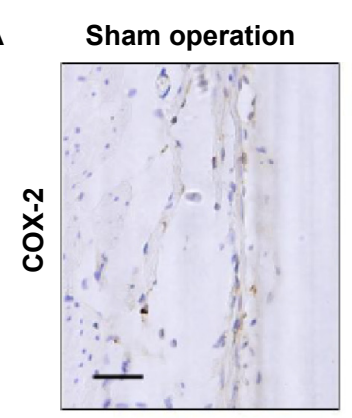

B

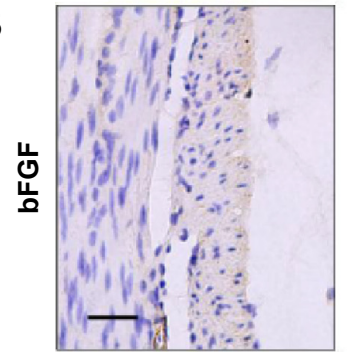

C

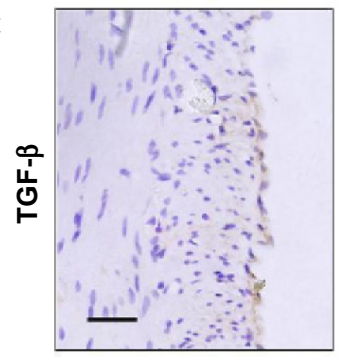

D

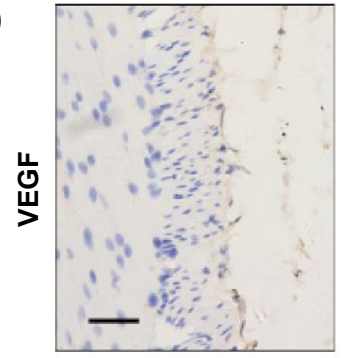

E

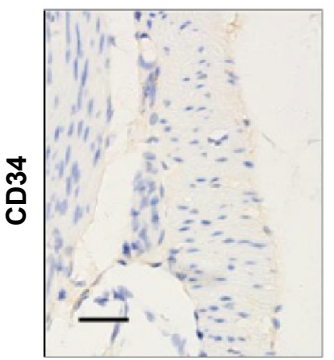

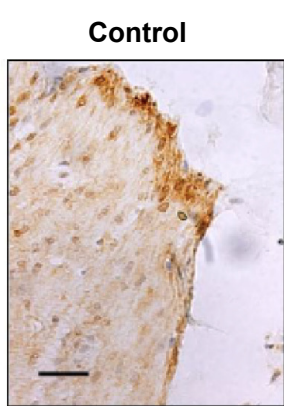
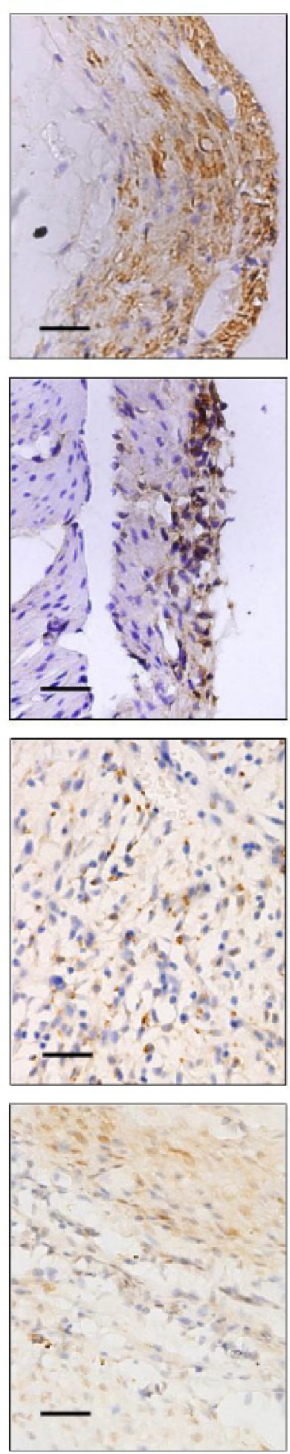
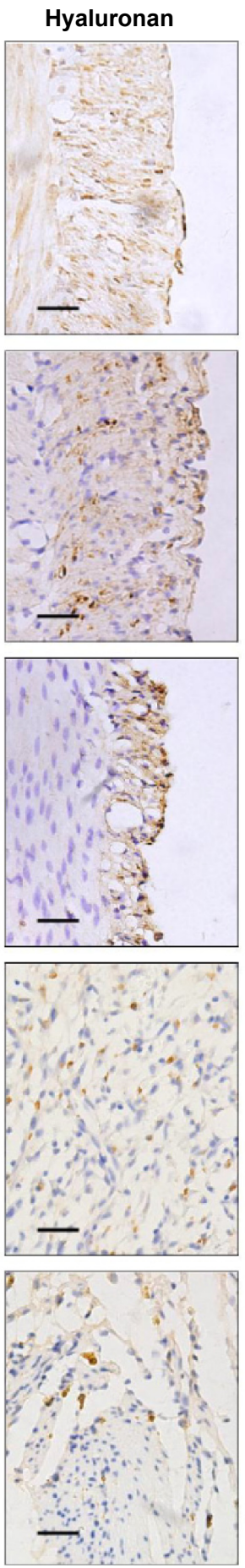
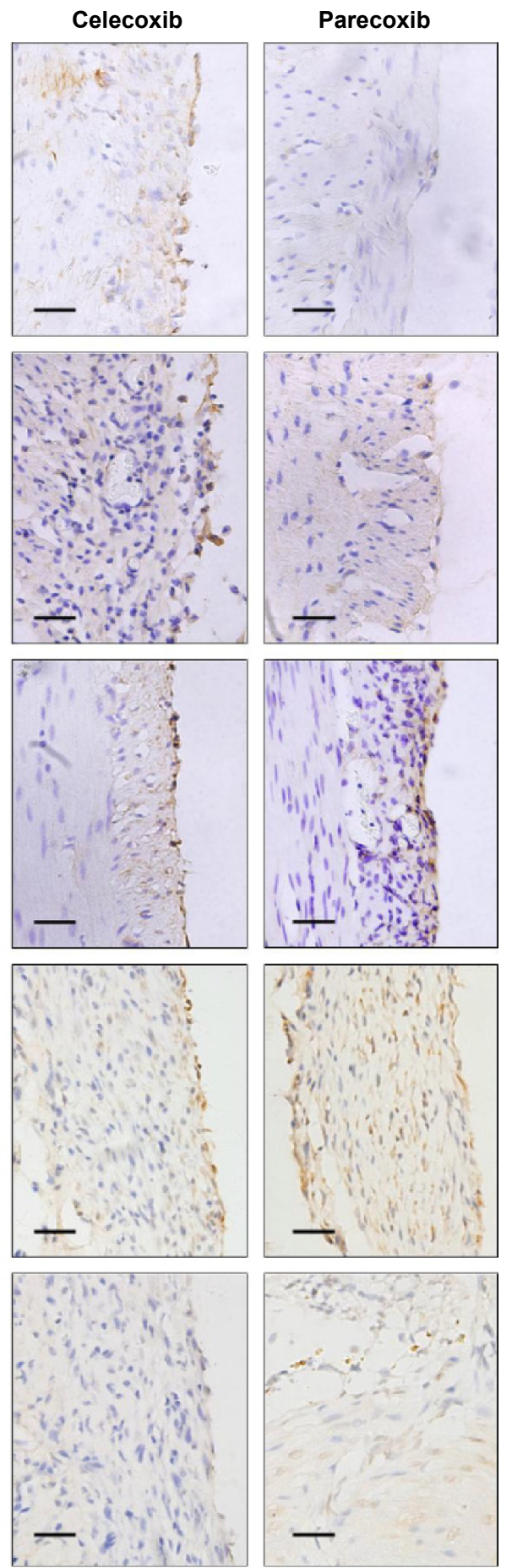

Figure 6 Immunohistochemical analysis of cyclooxygenase (COX)-2 (A), basic fibroblast growth factor (bFGF) (B), transforming growth factor-beta (TGF- $\beta$ ) (C), vascular endothelial growth factor (VEGF) (D), and cluster of differentiation (CD) 34 (E) in intra-abdominal adhesion tissues from groups that underwent peritoneum and cecum abrasion, and normal peritoneum in the sham operation group. Notes: Magnification 200x; scale bars, $25 \mu \mathrm{m}$.

Compared to normal peritoneum in the sham operation group, the adhesion tissues in the control group were well vascularized, containing a large number of microvessels (Figure 6E). The microvessel density in the hyaluronan treatment group was not different to that in the control animals.
The microvessel density in the celecoxib-treated, but not the parecoxib-treated, mice was less than that in the control mice, in spite of the significant effect of parecoxib on prevention of intra-abdominal adhesion formation and inhibition of bFGF and TGF- $\beta$ expression (Figure 6E). 
A

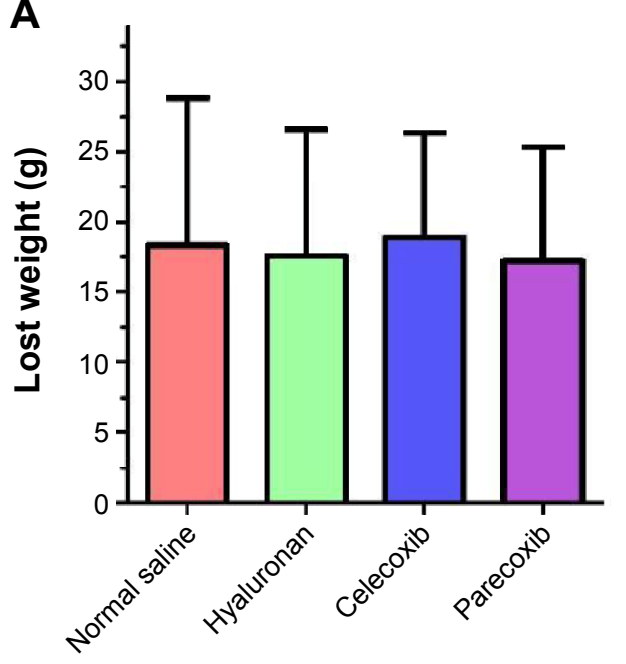

C

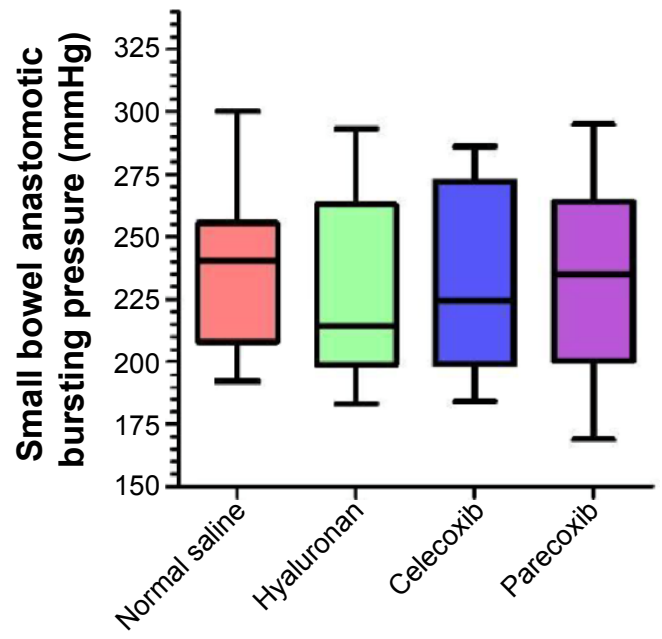

B
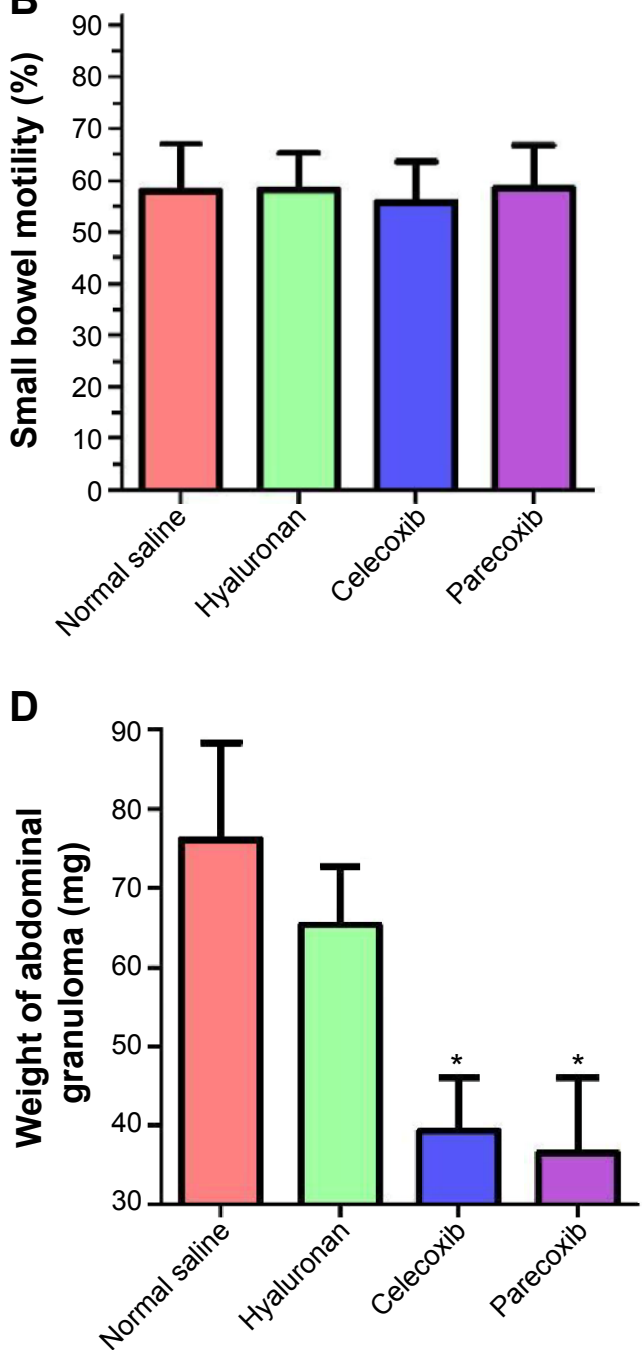

Figure 7 Use of selective cyclooxygenase (COX)-2 inhibitors to prevent intra-abdominal adhesion did not contribute to postoperative complications. Notes: The effect of selective COX-2 inhibitors on (A) animal weight loss, (B) small bowel motility, (C) small intestinal anastomoses, and (D) cotton pellet granuloma formation. $* P<0.05$, compared with the control group.

\section{Selective COX-2 inhibitors prevented intra-abdominal adhesion, but not postoperative complications}

The effects of two selective COX-2 inhibitors on postoperative recovery and inflammation were observed in a rat intestinal anastomosis model. No deaths or abdominal wound complications were observed. Weight loss in all rats was assessed on day 7 before the animals were euthanized; there was no difference between the four groups (Figure 7A). Thirty minutes before sacrifice, all animals were gavaged with charcoal. The propelled distance of the charcoal within the gastrointestinal tract in the mice was measured. As shown in Figure $7 \mathrm{~B}$, there was no difference in the propulsive function of the gastrointestinal tract between the groups. Additionally, the intestinal anastomotic bursting pressures shown in Figure 7C were not significantly different between the four groups. Both of the two selective COX-2 inhibitors, especially parecoxib, significantly decreased granuloma weight (approximately 50\%) compared to that of the normal saline group $(P<0.05)$ and the hyaluronan group $(P<0.05)$ (Figure 7D). However, there was no significant difference in granuloma weight between the normal saline group and the hyaluronan group $(P>0.05)$. These results indicate that inhibition of COX-2 may decrease inflammation, but does not affect postoperative recovery.

\section{Discussion}

In the current study, it was shown that hypoxia-induced COX-2 expression in peritoneal fibroblasts was involved in postoperative intra-abdominal adhesion formation. Inhibition 
of COX-2 resulted in decreased intra-abdominal adhesions in vivo. The data are consistent with the expression of COX-2 observed in adhesion fibroblasts. ${ }^{33}$ Treatment with selective COX-2 inhibitor has been shown previously to reduce the formation of postoperative adhesions in a rat model. ${ }^{15,34}$ However, in the current study, it was further shown that in vitro COX-2 inhibition decreased the effect of hypoxic activation on fibroblasts from the normal peritoneum. We provide evidence that the effect of selective COX-2 inhibitors on prevention of intra-abdominal adhesion possibly results from inhibition of bFGF and TGF- $\beta$ expression, but not through an antiangiogenic mechanism. Moreover, the use of selective COX-2 inhibitors to prevent intra-abdominal adhesion did not result in postoperative complications in the rat model.

Hypoxia, resulting from tissue injury, has been suggested to play an important role in wound healing, ${ }^{35,36}$ and may directly participate in the development of postoperative adhesions. ${ }^{37}$ Previous in vitro and in vivo studies ${ }^{12,21}$ have documented the gross and histological changes that may be triggered by hypoxia in peritoneal wounds. Moreover, it has been reported that exposure of normal peritoneal fibroblasts to hypoxia irreversibly induces TGF- $\beta 1$ and type I collagen to levels seen in adhesion fibroblasts. ${ }^{21}$ Here, we present compelling evidence that abnormal COX-2 expression in adhesive tissue via a hypoxia-dependent manner results in fibroblast activation.

COX-2 expression increases in response to various types of tissue injury, resulting in elevated levels of local PGs. ${ }^{38}$ Peritoneal tissue damage during operations can result in induction of $\mathrm{COX}-2$ expression during the healing process, with subsequent persistence of COX-2 expression by adhesion fibroblasts. ${ }^{33}$ The current results show that exposure of normal peritoneal fibroblasts to hypoxia significantly increases COX-2 expression and the enzymatic activity product $\mathrm{PGE}_{2}$. Based on previously published evidence and the current findings, we speculate that inhibition of COX-2 results in reversal of the activation of normal peritoneal fibroblasts in response to hypoxia, which may help prevent adhesion formation. This notion is further supported by the fact that the genetic or pharmacologic inhibition of COX-2 by shRNA or selective COX-2 inhibitors, respectively, decreased activation of normal peritoneal fibroblasts under hypoxia, suggesting that inhibition of COX-2 may be a potential target for the prevention of intra-abdominal adhesions.

A large amount of evidence ${ }^{39,40}$ has demonstrated that COX-2 is involved in pathologic inflammation and angiogenesis in various diseases. A previous report ${ }^{15}$ claimed that
COX-2 inhibitors, through their antiangiogenic properties, inhibit intra-abdominal adhesions in an experimental murine model. In the current study, it was found that, although both COX-2 inhibitors (celecoxib and parecoxib) have anti-adhesion properties, the VEGF levels and microvessel densities were unchanged in the parecoxib-treated group compared to in the control group. The inflammatory factors bFGF and TGF- $\beta$ were significantly decreased in response to COX-2 inhibition. Hence, we conclude that COX-2 inhibition prevents postoperative intra-abdominal adhesions by attenuating hypoxia-induced activation of peritoneal fibroblasts rather than anti-angiogenesis. Because wound healing is dependent on angiogenesis, there previously existed awareness that agents with antiangiogenic effect have been precluded as anti-adhesion agents because of their possibly negative effects on wound healing. ${ }^{16,19}$ Our results may help to eliminate this doubt of the inhibition of COX-2 as a prevention strategy for adhesions.

Parecoxib, which is a water soluble and injectable prodrug of valdecoxib, ${ }^{41}$ can be used perioperatively when patients are unable to take oral medications. Because inhibition of postoperative intestinal motility can result in an increased number of adhesions, ${ }^{42}$ special attention was paid to small bowel motility and intestinal anastomotic healing in the experiment animal; it was found that there was no adverse effect of parecoxib on post-operation recovery. Furthermore, parecoxib is approved through much of Europe for short-term perioperative pain control. ${ }^{43}$ Thus, we believe the COX-2 inhibitor parecoxib is a safe medicine for preventing the formation of postoperative adhesions.

\section{Limitations and future directions}

Unfortunately, the results of this study are not robust and should be further validated. A major weakness of this in vivo study is its reliance on one small animal model of adhesion. Moreover, there is a lack of accurate methods to evaluate severity of adhesions. Clinical trials need to be performed to confirm the beneficial properties and suitable dose of parecoxib in reducing adhesions.

\section{Conclusion}

We found that hypoxia-induced COX-2 expression in peritoneal fibroblasts is involved in postoperative intra-abdominal adhesion formation. The current results suggest that COX-2 inhibition can prevent postoperative intra-abdominal adhesions by decreasing hypoxia-induced activation of peritoneal fibroblasts rather than by anti-angiogenesis. This study indicates that inhibition of $\mathrm{COX}-2$ may represent a promising 
preventive strategy for postoperative intra-abdominal adhesions.

\section{Acknowledgments}

This study was supported by the National Natural Science Foundation of China (number 81201824), the Scientific and Technological Development Research Project Foundation by Shaanxi Province (number 2007K12-02), and the Fundamental Research Funds for the Central Universities in Xi'an Jiaotong University (number 2013jdhz33). We thank Medjaden Bioscience Limited for assisting in the preparation of this manuscript.

\section{Disclosure}

The authors declare no conflicts of interest in this work.

\section{References}

1. ten Broek RP, Issa Y, van Santbrink EJ, et al. Burden of adhesions in abdominal and pelvic surgery: systematic review and met-analysis. BMJ. 2013;347:f5588.

2. Fevang BT, Fevang J, Lie SA, Søreide O, Svanes K, Viste A. Longterm prognosis after operation for adhesive small bowel obstruction. Ann Surg. 2004;240(2):193-201.

3. Duron JJ, Silva NJ, du Montcel ST, et al. Adhesive postoperative small bowel obstruction: incidence and risk factors of recurrence after surgical treatment: a multicenter prospective study. Ann Surg. 2006;244(5): 750-757.

4. Mavros MN, Velmahos GC, Lee J, Larentzakis A, Kaafarani HM. Morbidity related to concomitant adhesions in abdominal surgery. J Surg Res. 2014;192(2):286-292.

5. Okabayashi K, Ashrafian H, Zacharakis E, et al. Adhesions after abdominal surgery: a systematic review of the incidence, distribution and severity. Surg Today. 2014;44(3):405-420.

6. Arung W, Meurisse M, Detry O. Pathophysiology and prevention of postoperative peritoneal adhesions. World J Gastroenterol. 2011; 17(41):4545-4553.

7. Diamond MP, Wexner SD, diZereg GS, et al. Adhesion prevention and reduction: current status and future recommendations of a multinational interdisciplinary consensus conference. Surg Innov. 2010;17(3): 183-188.

8. Ward BC, Panitch A. Abdominal adhesions: current and novel therapies. J Surg Res. 2011;165(1):91-111.

9. Wang N, Li Q, Zhang L, et al. Mesenchymal stem cells attenuate peritoneal injury through secretion of TSG-6. PLoS One. 2012;7(8):e43768.

10. Elliott CG, Wang J, Guo X, et al. Periostin modulates myofibroblast differentiation during full-thickness cutaneous wound repair. J Cell Sci. 2012;125(Pt 1):121-132.

11. Reijnen MM, Bleichrodt RP, van Goor H. Pathophysiology of intraabdominal adhesion and abscess formation, and the effect of hyaluronan. Br J Surg. 2003;90(5):533-541.

12. Ambler DR, Fletcher NM, Diamond MP, Saed GM. Effects of hypoxia on the expression of inflammatory markers IL-6 and TNF-a in human normal peritoneal and adhesion fibroblasts. Syst Biol Reprod Med. 2012; 58(6):324-329.

13. Saed GM, Zhang W, Diamond MP. Molecular characterization of fibroblasts isolated from human peritoneum and adhesions. Fertil Steril. 2001;75(4):763-768.

14. Saed GM, Munkarah AR, Abu-Soud HM, Diamond MP. Hypoxia upregulates cyclooxygenase-2 and prostaglandin E(2) levels in human peritoneal fibroblasts. Fertil Steril. 2005;83 Suppl 1:1216-1219.
15. Greene AK, Alwayn IP, Nose V, et al. Prevention of intra-abdominal adhesions using the antiangiogenic COX-2 inhibitor celecoxib. Ann Surg. 2005;242(1):140-146.

16. Cahill RA. Prevention of intra-abdominal adhesions using the antiangiogenic COX-2 inhibitor celecoxib. Ann Surg. 2006;244(2): 327-328; author reply 328.

17. Kim YI. Comparative study for preventive effects of intra-abdominal adhesion using cyclo-oxygenase-2 enzyme (COX-2) inhibitor, low molecular weight heparin (LMWH), and synthetic barrier. Yonsei Med J. 2013;54(6):1491-1497.

18. Arung W, Jehaes F, Cheramy JP, et al. Effects of parecoxib on the prevention of postoperative peritoneal adhesions in rats. J Invest Surg. 2013;26(6):340-346.

19. Cahill RA, Sheehan KM, Scanlon RW, Murray FE, Kay EW, Redmond HP. Effects of a selective cyclo-oxygenase 2 inhibitor on colonic anastomotic and skin wound integrity. Br J Surg. 2004;91(12): $1613-1618$

20. Saed GM, Kruger M, Diamond MP. Expression of transforming growth factor-beta and extracellular matrix by human peritoneal mesothelial cells and by fibroblasts from normal peritoneum and adhesions: effect of Tisseel. Wound Repair Regen. 2004;12(5):557-564.

21. Fletcher NM, Jiang ZL, Diamond MP, Abu-Soud HM, Saed GM. Hypoxia-generated superoxide induces the development of the adhesion phenotype. Free Radic Biol Med. 2008;45(4):530-536.

22. Paulissen SM, van Hamburg JP, Davelaar N, Asmawidjaja PS, Hazes JM, Lubberts E. Synovial fibroblasts directly induce Th17 pathogenicity via the cyclooxygenase/prostaglandin E2 pathway, independent of IL-23. J Immunol. 2013;191(3):1364-1372.

23. Wiktorowska-Owczarek A. The effect of valdecoxib on the production of growth factors evoked by hypoxia and bacterial lipopolysaccharide in HMEC-1 cells. Adv Clin Exp Med. 2013;22(6):795-800.

24. Lei J, Huo X, Duan W, et al. $\alpha$-Mangostin inhibits hypoxia-driven ROS-induced PSC activation and pancreatic cancer cell invasion. Cancer Lett. 2014;347(1):129-138.

25. Li X, Wang Z, Ma Q, et al. Sonic hedgehog paracrine signaling activates stromal cells to promote perineural invasion in pancreatic cancer. Clin Cancer Res. 2014;20(16):4326-4338.

26. Schmittgen TD, Livak KJ. Analyzing real-time PCR data by the comparative C(T) method. Nat Protoc. 2008;3(6):1101-1108.

27. Li X, Ma Q, Xu Q, et al. SDF-1/CXCR4 signaling induces pancreatic cancer cell invasion and epithelial-mesenchymal transition in vitro through non-canonical activation of Hedgehog pathway. Cancer Lett. 2012;322(2):169-176.

28. Peyton CC, Keys T, Tomblyn S, et al. Halofuginone infused keratin hydrogel attenuates adhesions in a rodent cecal abrasion model. J Surg Res. 2012;178(2):545-552.

29. Schuh-Hofer S, Tayefeh M, Reuter U, Dirnagl U, Arnold G. Effects of parecoxib on plasma protein extravasation and c-fos expression in the rat. Headache. 2006;46(2):276-285.

30. Katada J, Saito H, Ohashi A. Significance of cyclooxygenase-2 induced via p38 mitogen-activated protein kinase in mechanical stimulusinduced peritoneal adhesion in mice. J Pharmacol Exp Ther. 2005; 313(1):286-292.

31. Aarons CB, Cohen PA, Gower A, et al. Statins (HMG-CoA reductase inhibitors) decrease postoperative adhesions by increasing peritoneal fibrinolytic activity. Ann Surg. 2007;245(2):176-184.

32. Fu F, Hou Y, Jiang W, Wang R, Liu K. Escin: inhibiting inflammation and promoting gastrointestinal transit to attenuate formation of postoperative adhesions. World J Surg. 2005;29(12):1614-1620; discussion 1621-1622.

33. Saed GM, Munkarah AR, Diamond MP. Cyclooxygenase- 2 is expressed in human fibroblasts isolated from intraperitoneal adhesions but not from normal peritoneal tissues. Fertil Steril. 2003;79(6): 1404-1408.

34. Guvenal T, Cetin A, Ozdemir H, Yanar O, Kaya T. Prevention of postoperative adhesion formation in rat uterine horn model by nimesulide: a selective COX-2 inhibitor. Hum Reprod. 2001;16(8):1732-1735. 
35. Ruthenborg RJ, Ban JJ, Wazir A, Takeda N, Kim JW. Regulation of wound healing and fibrosis by hypoxia and hypoxia-inducible factor-1. Mol Cells. 2014;37(9):637-643.

36. Lokmic Z, Musyoka J, Hewitson TD, Darby IA. Hypoxia and hypoxia signaling in tissue repair and fibrosis. Int Rev Cell Mol Biol. 2012; 296: 139-185.

37. Saed GM, Diamond MP. Effects of interferon-gamma reverse hypoxiastimulated extracellular matrix expression in human peritoneal and adhesion fibroblasts. Fertil Steril. 2006;85 Suppl 1:1300-1305.

38. Cesario A, Rocca B, Rutella S. The interplay between indoleamine 2,3-dioxygenase 1 (IDO1) and cyclooxygenase (COX)-2 in chronic inflammation and cancer. Curr Med Chem. 2011;18(15):2263-2271.

39. Salvado MD, Alfranca A, Haeggström JZ, Redondo JM. Prostanoids in tumor angiogenesis: therapeutic intervention beyond COX-2. Trends Mol Med. 2012;18(4):233-243.
40. Alhouayek M, Muccioli GG. COX-2-derived endocannabinoid metabolites as novel inflammatory mediators. Trends Pharmacol Sci. 2014; 35(6):284-292.

41. Hullett B, Salman S, O'Halloran SJ, Peirce D, Davies K, Ilett KF. Development of a population pharmacokinetic model for parecoxib and its active metabolite valdecoxib after parenteral parecoxib administration in children. Anesthesiology. 2012;116(5):1124-1133.

42. Ouaïssi M, Gaujoux S, Veyrie N, et al. Post-operative adhesions after digestive surgery: their incidence and prevention: review of the literature. J Visc Surg. 2012;149(2):e104-e114.

43. Athanasakis K, Petrakis I, Vitsou E, Pimenidou A, Kyriopoulos J. A cost-effectiveness analysis of parecoxib in the management of postoperative pain in the Greek health care setting. Clin Ther. 2013;35(8) 1118-1124. 


\section{Supplementary material}

Table SI Real-time PCR primer sequence

\begin{tabular}{|c|c|}
\hline Genes & Primer sequence \\
\hline \multirow[t]{2}{*}{ Human COX-2 } & PI: 5'-AGGACGGCAATGCTGATG-3' \\
\hline & P2: 5'-TCGTAGTTGGCGGTCGTG-3' \\
\hline Human GAPDH & PI: 5'-ACCACAGTCCATGCCATCAC-3' \\
\hline Rat GAPDH & P2: 5'-TCCACCACCCTGTTGCTGTA-3' \\
\hline \multirow[t]{2}{*}{ Rat COX-2 } & PI: 5'-CACGGACTTGCTCACTTTGTT-3' \\
\hline & P2: 5'-AAGCGTTTGCGGTACTCATT-3' \\
\hline \multirow[t]{2}{*}{ Rat $\alpha-S M A$} & PI: 5'-CCGGGAGAAAATGACTCAAA-3' \\
\hline & P2: 5'-GCGTCCAGAGGCATAGAGAG-3' \\
\hline \multirow[t]{2}{*}{ Rat type I collagen ( $|a|)$} & PI: 5'-CATCGGTGGTACTAAC-3' \\
\hline & P2: 5'-CTGGATCATATTGCACA-3' \\
\hline
\end{tabular}

\section{Publish your work in this journal}

Drug Design, Development and Therapy is an international, peerreviewed open-access journal that spans the spectrum of drug design and development through to clinical applications. Clinical outcomes, patient safety, and programs for the development and effective, safe, and sustained use of medicines are a feature of the journal, which has also been accepted for indexing on PubMed Central. The manuscript management system is completely online and includes a very quick and fair peer-review system, which is all easy to use. Visit http://www.dovepress.com/testimonials.php to read real quotes from published authors.

Submit your manuscript here: http://www.dovepress.com/drug-design-development-and-therapy-journal 